\title{
An improved method of microscopic image segmentation
}

\author{
Zhang Feng Shou ${ }^{1}$,Dong Fang ${ }^{1}$,Liu Jian Ting ${ }^{1}$ and Meng Xin ${ }^{1}$ \\ ${ }^{1}$ Henan university of science and technology,HeNan471023, China.
}

\begin{abstract}
In order to improve the effectiveness and accuracy of image processing in modern medical inspection, a segmentation image optimization algorithm of improved two-dimensional maximum entropy threshold based on genetic algorithm combined with mathematical morphology is proposed, in view of the microscopic cell images characteristic and the shortcomings of the traditional segmentation algorithm. Through theoretical analysis and contrast test, the segmentation method proposed is superior to the traditional threshold segmentation method in microscopic cell images, and the average segmentation time of the improved algorithm is $73 \%$ and $44 \%$ higher than the traditional two-dimensional maximum entropy threshold and the improved two-dimensional maximum entropy threshold.
\end{abstract}

\section{INTRODUCTION}

Image segmentation is the key step of the process from image processing to image analysis[1-3]. The background of high noise microscopic images presents great complexity, therefore, it is difficult to simultaneously extract the object contours possible during filtering image noise by a single image segmentation method[4-5]. In this paper, we would analyze the traditional segmentation algorithm from experiment sand put forward an improved genetic algorithm of threshold segment method by using two-dimensional maximum entropy. Then, the coarse segmentation of microscopic images are presented.Finally,the object contoury extracted after filtering out residual noise and filling small holes in the target by mathematical morphology methods.

\section{IMPROVED METHOD}

\subsection{Improved two-dimensional maximum entropy threshold based on genetic algorithm}

The two-dimensional maximum entropy threshold algorithm through the concept of entropy in information theory combines the point gray and the region gray. For low signal-to-noise ratio images,using two-dimensional maximum entropy threshold method can obtain a better image segmentation results[6-8]. The advantage of the traditional two-dimensional maximum entropy threshold algorithm is that it can reduce the digital image sensitivity to noise,but this algorithm is a time-consuming operation and high computational complexity. The gray histogram with an improved two-dimensional maximum entropy threshold algorithm, as shown in Figure 1.

Let the microscopic image be $f(x, y)$ and the gray series be L. Another microscopic image $\mathrm{g}(\mathrm{x}, \mathrm{y})$ is obtained by adopting neighborhood average to original image, whose gray scale is still L.Image sizeis $\mathrm{M} \times \mathrm{N}$.Let nij be pixels of $\mathrm{f}(\mathrm{x}, \mathrm{y})$ in gray value $\mathrm{i}$ and $\mathrm{g}(\mathrm{x}, \mathrm{y})$ in the gray value of $j$. The probability of the occurrence of $(i, j)$ of the point grey area and the region gray is flowing.

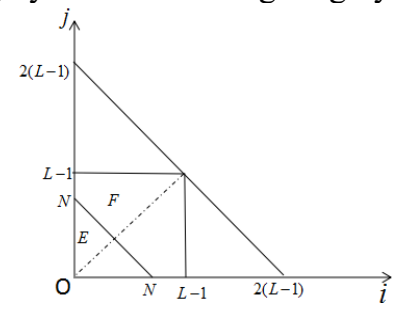

Fig. 1 Improved two-dimensional gray histogram of image

In the method the sum of the pixel gray values and the neighbor average grey values $\mathrm{N}$ is the independent variable, pixels smaller than $\mathrm{N}$ are divided into one class, and pixels larger than $\mathrm{N}$ are divided into another class. The region $\mathrm{E}$ and region $\mathrm{F}$ respectively represent the target and background in the image,the calculation is reduced from two dimensions to one dimension by finding the best image segmentation pixel value. The processing effect equivalently is first smoothing the image and then segmenting it. The probability of the region $\mathrm{E}$ is as follows from the figure 1:

$$
P_{E}=\sum_{i=0}^{N-1} \sum_{j=o}^{N-1} p_{i j},(i, j \in E)
$$

The threshold selection criteria is $\mathrm{i}+\mathrm{j}=\mathrm{N}$, then the following equations can be obtained

$$
P_{E}(N)=P_{E}(N-1)+P_{0, N}+P_{1, N-1}+\cdots+P_{N, 0}
$$

\footnotetext{
*Corresponding author: \{Dong Fang ${ }^{\text {a }}$ fantasyericdong@163.com
} 


$$
\begin{aligned}
H_{E}(N)= & H_{E}(N-1)-\left(P_{0, N}+P_{1, N-1}+\cdots+P_{N, 0}\right) \\
& \times \lg \left(P_{0, N}+P_{1, N-1}+\cdots+P_{N, 0}\right) \\
H_{E}= & \ln P_{E}+\frac{H_{E}}{P_{E}}, H_{F}=\ln \left(1-P_{E}\right)+\frac{H_{L}-H_{E}}{1-P_{E}}
\end{aligned}
$$

The decision function of entropy is defined as

$$
\psi(N)=\max \left\{H_{E}+H_{F}\right\}
$$

Genetic algorithm is introduced based on all the above.An improved two-dimensional maximum entropy threshold genetic algorithm is proposed to further reduce computational complexity and shorten the computation time.

In this paper,the improved algorithm is based on the improved two-dimensional entropy threshold algorithm. The sum of the pixel gray values and the neighbor average grey values $\mathrm{N}$ is the independent variable, so $\mathrm{N}$ is the solution of the problem. Because the range of $\mathrm{N}$ is $0 \sim 511$, the 9-bit binary encoding is adopted when $\mathrm{N}$ values are encoded.The $\mathrm{N}$ value is encoded into a gene string $\mathrm{a}=\{\mathrm{a} 0, \cdots, \mathrm{a} 8\}$, In the formula, $a$ represents the value of $\mathrm{N}$.

The specific process is as follows:

(1)Initial population generation. The solution space is binary encoded and initial population is generated randomly. Population numbers generally take $20 \sim 200$.

(2)Determine fitness and fitness function.In this algorithm,equation (4) is fitness function. Its fitness is high when the gene string with a large entropy valuepresents the $\mathrm{N}$ value in the equation (4). On the contrary, its fitness is low.

(3)Selection action. The individual ai with the lowest fitness in the current population and the individual aj with the highest fitnessamong generations of populations are found by combining optimal preservation strategy and roulette wheel selection. The code value of aj is instead of ai encoding value,other individuals are selected by probability of roulette wheel selection. Thus, the diversity of the population is maintained while the optimal individual is not destroyed.

(4)Crossover and mutation operation. The crossover and mutation of the biological can make the species evolve rapidly or produce new species, but crossover and mutation operation will speed up the optimization. Crossover rate values generally take $0.4 \sim 0.99$. The original highly fitness gene string will be destructed due to crossover rate is too large, and the crossover rate is too small to keep the search process.Mutation ratevalues generally take $0.0001 \sim 0.1$. Genetic algorithm will turn into random search due to the mutation rate is too large, and mutation rate is too small to produce new individuals.

(5)Set criteria of termination calculate on. A practical algorithm must be convergent. In the genetic algorithm, the best gene string is returned when the maximum propagation algebra is achieved, and it is used as two-dimensional segmentation threshold. Then the algorithm is ending, otherwise, returning (2)and proceeding to the next generation.
After segmenting the microscopic images with animprovedtwo-dimensional maximum entropy threshold based on genetic algorithm, most of the noises are removed, but there is some small holes that goes on in target area and some undesired impurity appeared, so mathematical morphology methods are used to fill the small holes in the target area by multiple close calculation, and remove thin impurity by multiple open calculation. Finally, the object's contour is extracted.

\section{EXPERIMENT}

In order to verify the effectiveness of segmentation algorithm in this paper,three micrograph were used to experimentalize. The micrographs are BMP format and the resolution is $130 \times 130$. Experiment platform is $\operatorname{Intel}(\mathrm{R})$ Core(TM) CPU $3.4 \mathrm{GHz}$ and memory is $4 \mathrm{~GB}$, windows 7 operating system, the algorithm is implemented with VS2013+OpenCV3.0 compiler platform.

Microscopic cell original imageis as shown in figure 2.The traditional image segmentation algorithm is difficult to obtain satisfactory results. The first order edge operator Sobel and two order edge operator LoG are used in edge detection. The segmentation effect of microscopic cell images are shown in figure 3 .

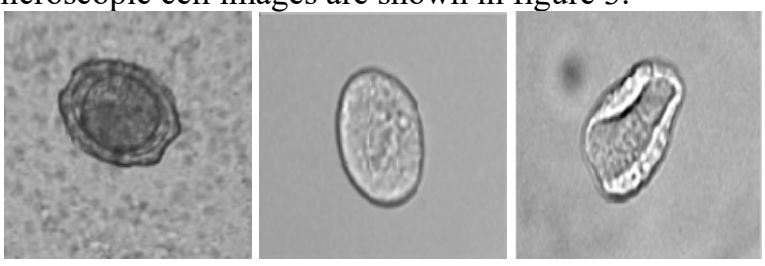

Fig. 2 Microscopic cell original image

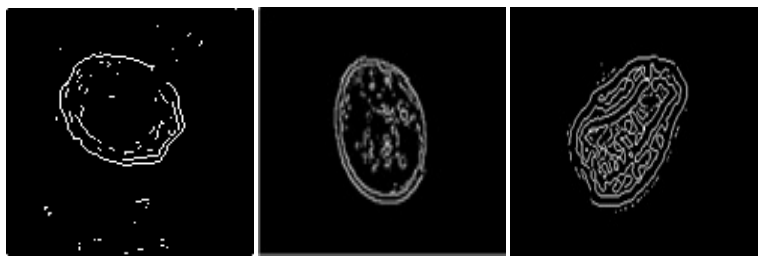

Fig. 3 Image processing of sobel edge detection

As can be seen from the figure 3, sobel edge operator is very sensitive to noise, which makes the target edge and noise benot distinguished.The noise is reduced after dealing with first order edge operators, but double edges and edge breaks are appearing. Therefore, the edge detection can not segment the high noise microscopic image very well.

According to the one-dimensional and two-dimensional gray histogram of microscopic images(Take the case ofmicroscopic image 1, as shown in Figure 4), and Otsu threshold method and two-dimensional maximum entropy threshold method were used to segment the microscopic images, the results are shown in Figure 5. 

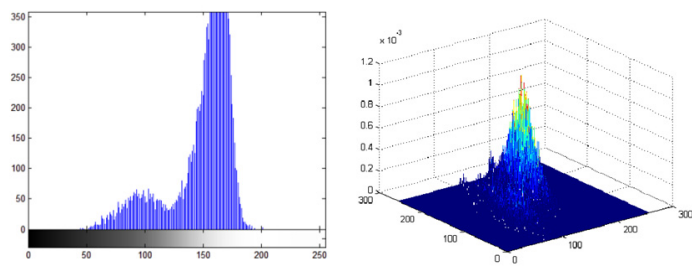

Fig. 4 Grey level histogram
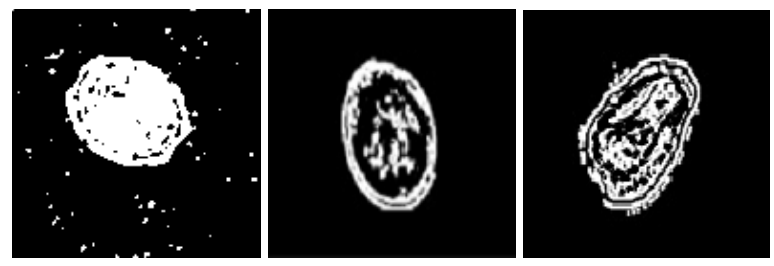

Otsuthreshold segmentation
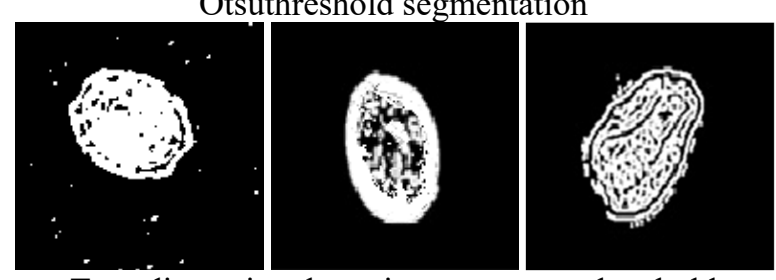

Two-dimensional maximum entropy threshold segmentation

Fig. 5 Threshold segmentation

The figure 5 shown that the traditional threshold segmentation method is poorly segmented the edges of microscopic images. The proposed method is used to segment the microscopic image, and the effect shown in Figure 6.
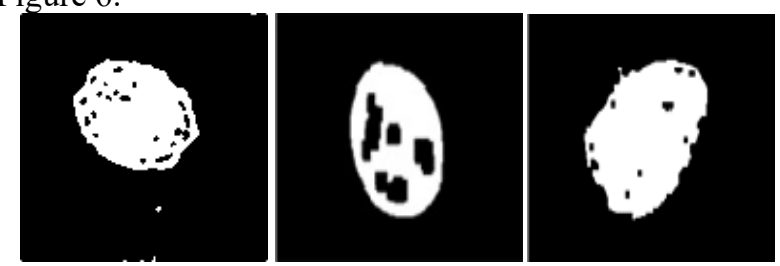

Improved two-dimensional maximum entropy threshold based on genetic algorithm:
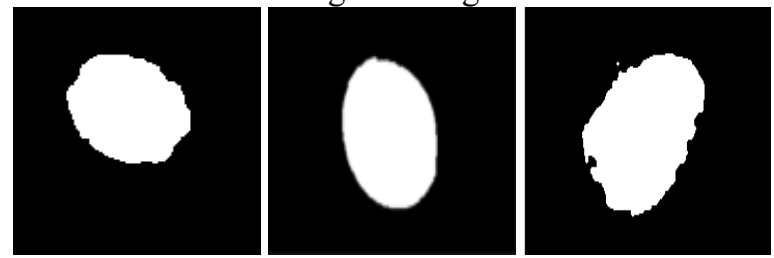

Mathematical morphology filtering

Fig. 6 Segmentation method in this paper

In the improved two-dimensional maximum entropy threshold genetic algorithm, the initial population $\mathrm{N}$ is 30 , the chromosome has a coding length of 9 bits, the crossover rate is 0.5 , the mutation rate is 0.05 . After 8 generations, the inheritance converges to the optimum threshold.

Tab.1 The segmentation time comparison (unit:s)

\begin{tabular}{|c|l|l|l|}
\hline $\begin{array}{c}\text { Microscopic cell } \\
\text { image }\end{array}$ & image1 & image2 & image3 \\
\hline
\end{tabular}

\begin{tabular}{|c|c|c|c|}
\hline $\begin{array}{c}\text { Traditional 2D } \\
\text { optimal entropy } \\
\text { threshold algorithm }\end{array}$ & 0.704 & 0.678 & 0.768 \\
\hline $\begin{array}{c}\text { Improved optimal } \\
\text { entropy threshold } \\
\text { algorithm }\end{array}$ & 0.313 & 0.389 & 0.501 \\
\hline $\begin{array}{c}\text { The improved } \\
\text { algorithm }\end{array}$ & 0.202 & 0.258 & 0.241 \\
\hline
\end{tabular}

It can be concluded from Figure 6 and that the object contour is intact, the inner wormhole disappears and the noise in the background is completely removed. Besides, an ideal contour two-value image is obtained. The algorithm operation speed is shown Table 1. From the Table, the average segmentation time of the improved algorithm is $73 \%$ higher than the traditional two-dimensional maximum entropy threshold and it is $44 \%$ higher than the improved two-dimensional maximum entropy threshold.

\section{CONCLUSION}

In this paper, an optimization algorithm of improved two-dimensional maximum entropy threshold genetic algorithm based rough segmentation combined with morphology fine segmentation image is proposed. The effect of segmentation microscopic image is very good by the improved optimization algorithm. The result is that the object contour is intact, the inner wormhole disappears and the noise in the background is completely removed.Besides, an ideal microscopic cell contour two-value image was obtained. Through theoretical analysis and contrast test, the segmentation method proposed is superior to the traditional threshold segmentation method in microscopic cell images, and achieve ideal segmentation results, the segmentation accuracy and speed is superior to the traditional threshold segmentation method.

\section{REFERENCES}

1. YANG Xiaoqing. Microscopic cell image processing and application based on morphology[J]. Computer Systems \& Applications, Vol.2016,(03):p.220-224.

2. Du Peijun. Two dimensional maximum entropy image segmentation based on artificial bee colony optimization[J]. Computer Engineering, 2012,(09):223-225+243.

3. QIAO Lingling, MAO xiaoju. Otsu image segmentation method based on improved genetic algorithm[J]. Journal of Changchun Institute of Technology(Natural Sciences Edition), Vol. 2016,(04):p.105-107.

4. Melkemi K E,MohamedB,Sebti F.A multiagent system approach for image segmentation using genetic algorithms and extremal optimization heuristics[J]. Pattern Recognition Letters, 2016, 27(11):p.1230-1238. 
5. TUO Qian. Study of Image thresholding segmentation algorithm on maximum entropy combining genetic algorithm[D]. Kunming University of Science and Technology,2016.

6. Shen Xiaoci. Research of two-dimensional entropy image segmentation method based on genetic algorithm[D]. China University of Geosciences (Beijing) ,2008.

7. Zhao Song, XIA Yanling. Improvement of DNA genetic algorithm two-dimensional maximum entropy image segmentation[J]. Silicon Valley, 2013,(02):250-252+211.

8. PENG Jing, ZHANG Baoge. A two-dimensional maximum entropy segmentation algorithm based on boundary feature $[\mathrm{J}]$. Computer and Digital Engineering, 2008,(07):18-20. 